\title{
The effect of paired corticospinal-motoneuronal stimulation on maximal voluntary elbow flexion in cervical spinal cord injury: an experimental study
}

\author{
Siobhan C. Dongés ${ }^{1} \cdot$ Claire L. Boswell-Ruys ${ }^{1,2,3} \cdot$ Jane E. Butler $^{1,2} \cdot$ Janet L. Taylor ${ }^{1,2,4}$
}

Received: 21 December 2018 / Accepted: 17 April 2019 / Published online: 13 May 2019

(c) International Spinal Cord Society 2019

\begin{abstract}
Study design Randomised, controlled, crossover study.

Objectives Paired corticospinal-motoneuronal stimulation (PCMS) involves repeatedly pairing stimuli to corticospinal neurones and motoneurones to induce changes in corticospinal transmission. Here, we examined whether PCMS could enhance maximal voluntary elbow flexion in people with cervical spinal cord injury.

Setting Neuroscience Research Australia, Sydney, Australia.

Methods PCMS comprised 100 pairs of transcranial magnetic and electrical peripheral nerve stimulation $(0.1 \mathrm{~Hz})$, timed so corticospinal potentials arrived at corticospinal-motoneuronal synapses $1.5 \mathrm{~ms}$ before antidromic motoneuronal potentials. On two separate days, sets of five maximal elbow flexions were performed by 11 individuals with weak elbow flexors post C4 or C5 spinal cord injury before and after PCMS or control (100 peripheral nerve stimuli) conditioning. During contractions, supramaximal biceps brachii stimulation elicited superimposed twitches, which were expressed as a proportion of resting twitches to give maximal voluntary activation. Maximal torque and electromyographic activity were also assessed. Results Baseline median (range) maximal torque was $11 \mathrm{Nm}(6-41 \mathrm{Nm})$ and voluntary activation was $92 \%$ (62-99\%). Linear mixed modelling revealed no significant differences between PCMS and control protocols after conditioning (maximal torque: $p=0.87$, superimposed twitch: $p=0.87$, resting twitch: $p=0.44$, voluntary activation: $p=0.36$, biceps EMG: $p=0.25$, brachioradialis EMG: 0.67).

Conclusions Possible explanations for the lack of effect include a potential ceiling effect for voluntary activation, or that PCMS may be less effective for elbow flexors than distal muscles. Despite results, previous studies suggest that PCMS is worthy of further investigation.
\end{abstract}

\section{Introduction}

Corticospinal pathway damage caused by spinal cord injury can result in persistent reductions in descending drive to

Supplementary information The online version of this article (https:// doi.org/10.1038/s41393-019-0291-3) contains supplementary material, which is available to authorized users.

\footnotetext{
Janet L. Taylor

janet.taylor@ecu.edu.au

Neuroscience Research Australia, Sydney, Australia

2 University of New South Wales, Sydney, Australia

3 Prince of Wales Hospital, Sydney, Australia

4 Edith Cowan University, Perth, Australia
}

muscles and impaired voluntary motor output. However, the corticospinal pathway is capable of plasticity and reorganisation after injury, and changes are associated with functional improvements after training and rehabilitation [1]. In humans, the corticospinal pathway has a monosynaptic component, where a portion of corticospinal axons arising from the primary motor cortex synapse onto spinal motoneurones, allowing direct voluntary control of motoneuronal output to muscles [2-4]. Techniques targeting remaining monosynaptic connections after incomplete spinal cord injury may enhance corticospinal transmission and improve motor control.

Synaptic modification can be induced with repeated pairs of presynaptic orthodromic and postsynaptic antidromic potentials, timed to arrive at the synapse within milliseconds of each other. Arrival of presynaptic potentials before postsynaptic tends to potentiate synapses, whereas 
the opposite tends to depress. These changes are known as spike-timing-dependent plasticity (STDP) [5]. STDP-like changes can be induced at corticospinal-motoneuronal connections using a technique that involves repeated pairs of single-pulse transcranial magnetic stimulation (TMS) and single-pulse peripheral nerve stimulation (PNS) at maximal intensities (eliciting antidromic activation of all motoneurones). This technique, known as paired corticospinal-motoneuronal stimulation (PCMS), can modify muscle responses to TMS and cervicomedullary stimulation, enhance voluntary motor output during lowforce contractions and improve hand dexterity after spinal cord injury [6-10]. In people without spinal cord injury, PCMS enhanced maximal voluntary activation (level of voluntary neural drive to the muscle during maximal efforts) in comparison with a TMS-only control protocol [11]. Twitch interpolation [12] was used to measure voluntary activation. This involves delivery of a supramaximal stimulus to the nerve supplying the muscle during a voluntary contraction to produce a force increment known as a superimposed twitch. To quantify voluntary activation the superimposed twitch is expressed as a proportion of a twitch elicited by the same stimulus at rest [13-15]. If a muscle had all motor units firing maximally, there would be no superimposed twitch. However, full activation is rare, even with maximal effort [15-18].

Maximal motor output is reduced after motor incomplete spinal cord injury [19-25], and PCMS can enhance maximal voluntary activation in people without spinal cord injury [11]. Thus, we hypothesised that PCMS would enhance voluntary activation and increase force and muscle activity during maximal elbow flexion in people with neurologically weak elbow flexors post cervical spinal cord injury.

\section{Methods}

\section{Participants}

Eleven individuals (aged 51y (12) (mean (SD)); 1F) with chronic ( $\geq 1 \mathrm{y}$ post injury) cervical spinal cord injury participated in the study (Table 1 for participant details). The tested arm was required to have some voluntary elbow flexion. If both sides were able, the weaker arm was chosen. Before commencing the study, participants underwent a five-point clinical strength assessment for elbow flexion [26], performed by the same qualified physiotherapist. All participants gave informed written consent.

\section{PCMS}

The experimental setup is described in Fig. 1a ( $\mathrm{S} 1$ for details). TMS (Magstim 200, Magstim, Whitland, UK) over the primary motor cortex elicited corticospinal volleys, and electrical PNS (DS7AH constant current stimulator, Digitimer, Welwyn Garden City, UK) at the brachial plexus elicited maximal compound muscle action potentials, implying antidromic activation of all motoneurones. Interstimulus interval (TMS before PNS; $2.7 \mathrm{~ms}$ (1.4)) was individually calculated so corticospinal volleys would arrive presynaptically at corticospinal-motoneuronal synapses $1.5 \mathrm{~ms}$ before antidromic, postsynaptic motoneurone volleys. For further details of TMS, PNS and interstimulus interval calculation see S2. PCMS consisted of 100 paired stimuli $(0.1 \mathrm{~Hz})$. This was compared with control conditioning of 100 PNS only performed on a separate day (control conditioning and PCMS day were randomly ordered) (Fig. 1b).

Table 1 Participant details

\begin{tabular}{llllllll}
\hline Participant & Age & $\begin{array}{l}\text { Single } \\
\text { neurological } \\
\text { level of injury }\end{array}$ & $\begin{array}{l}\text { Motor level } \\
\text { of injury } \\
\text { (tested arm) }\end{array}$ & $\begin{array}{l}\text { ASIA } \\
\text { impairment scale }\end{array}$ & $\begin{array}{l}\text { Time } \\
\text { since } \\
\text { injury (y) }\end{array}$ & Tested arm & $\begin{array}{l}\text { Clinical } \\
\text { strength } \\
\text { assessment }\end{array}$ \\
\hline 1 & 33 & C4 & C4 & C & 2 & R & 2 \\
2 & 23 & C4 & C4 & D & 2 & L & 2 \\
3 & 56 & C4 & C5 & A & 9 & L & 4 \\
4 & 54 & C5 & C5 & A & 31 & L & 4 \\
5 & 53 & C4 & C5 & B & 34 & L & 3 \\
6 & 60 & C4 & C5 & B & 2 & L & 4 \\
7 & 45 & C4 & C5 & C & 31 & R & 4 \\
8 & 57 & C4 & C5 & D & 1 & L & 4 \\
9 & 58 & C4 & C5 & D & 5 & L & 3 \\
10 & 61 & C4 & C5 & D & 2 & R & 3 \\
11 & 59 & C5 & C6 & B & 33 & L & 5
\end{tabular}

ASIA American Spinal Injury Association. Participants are numbered in order of motor level of injury (tested arm), and then ASIA impairment scale. Participant 3 is the only female participant. Note that although participant 11 tested at grade 5 in the clinical strength assessment, their maximal voluntary torque $(32.5 \mathrm{Nm})$ was greatly reduced in comparison with men without a spinal cord injury $(\sim 65-70 \mathrm{Nm})$ [18, 30-32] 


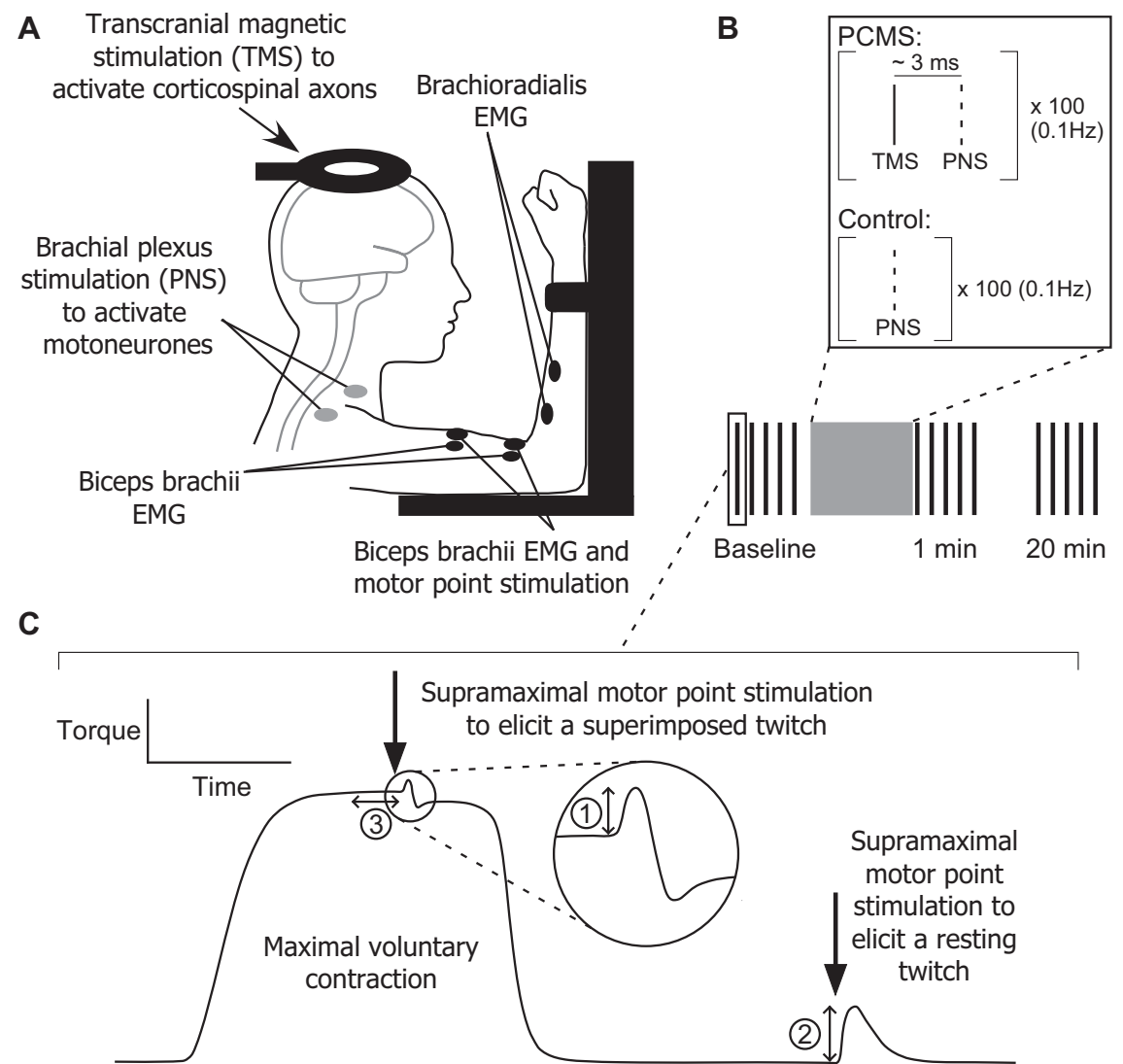

Fig. 1 Experimental setup and protocol. a Participants sat upright in their own wheelchair or a laboratory chair with the selected arm placed in an arm bar. The shoulder and elbow were flexed at 90 degrees and the forearm supinated. Transcranial magnetic stimulation (TMS) over the primary motor cortex elicited corticospinal volleys, and electrical peripheral nerve stimulation (PNS) at the brachial plexus elicited antidromic motoneuronal volleys. Supramaximal biceps brachii motor point stimulation was used to elicit twitches. Elbow torque was measured using a linear strain gauge coupled to the arm bar and EMG was recorded through surface electrodes placed over the muscle belly and distal tendon of each muscle. b One baseline set of five maximal voluntary elbow flexions ( 2 min between contractions) was performed

\section{Outcome measures}

\section{Motor point stimulation}

Single, supramaximal stimuli $(200-\mu$ s pulse width in 10 participants, $100 \mu \mathrm{s}$ in one participant to minimise triceps activation; DS7AH stimulator) were delivered to biceps through surface electrodes (see S1). On each day, stimulus intensity was increased until no further increase was seen in resting twitch torque, and $110 \%$ of this intensity (109 mA (42)) was used.

\section{Maximal voluntary contractions (MVCs)}

Before and after PCMS or control conditioning, participants performed brief ( 2-3 s), isometric MVCs of elbow flexors. before paired corticospinal-motoneuronal stimulation (PCMS) or control conditioning (PCMS: 100 TMS and PNS pairs at $0.1 \mathrm{~Hz}$, TMS $\sim 3$ ms prior to PNS; control: 100 PNS only, $0.1 \mathrm{~Hz}$ ). This was followed by two more sets of maximal voluntary elbow flexions $\sim 1$ min and $\sim 20$ min after conditioning. c An illustrative example of a maximal voluntary contraction. For each contraction, supramaximal motor point stimulation elicited (1) superimposed and (2) resting twitches. Twitch amplitudes were measured from twitch onset to peak, and maximal voluntary activation was calculated using the formula (1-superimposed twitch/resting twitch) $\times 100 \%$. (3) Maximal voluntary torque, and biceps and brachioradialis EMG were measured over the $100 \mathrm{~ms}$ prior to motor point stimulation during the maximal voluntary contraction

Participants performed two or three practice MVCs prior to data collection. An LED display provided visual feedback of torque, and participants received verbal encouragement. During each MVC, motor point stimulation elicited a superimposed twitch. Shortly after each MVC, when force returned to baseline, motor point stimulation elicited a potentiated resting twitch (Fig. 1c). Voluntary activation was calculated from twitch amplitudes. Other outcomes were maximal voluntary torque and EMG.

\section{Protocol}

The study had a randomised, controlled, crossover repeatedmeasures design. In random order, the effects of PCMS and control conditioning on maximal voluntary torque, twitch amplitude, voluntary activation, and EMG were examined 
on different days (5-22 d apart) (Fig. 1b). Session time was the same on both days for all participants except one (2-h difference in start-time). Participants performed one baseline set of five brief $(\sim 2-3 \mathrm{~s})$ MVCs, with 2 min between contractions to avoid fatigue. Then they underwent $\sim 16.5$ min conditioning (PCMS or control) during which they could watch a documentary. Two further MVC sets followed starting at 1-6 min and 15-20 min post-conditioning. This variability in timing was introduced by individual participant requirements.

\section{Data analyses and statistics}

\section{Analyses}

For each MVC set, baseline torque was measured as mean torque over $100 \mathrm{~ms}$ prior to motor point stimuli for all five resting twitches. Exclusion from baseline torque calculation occurred when visual EMG inspection revealed ongoing muscle activity before the resting twitch. Maximal voluntary torque during each MVC was measured as mean torque over $100 \mathrm{~ms}$ prior to motor point stimulation, minus baseline torque. Twitch amplitudes were measured from twitch onset to peak, and voluntary activation was calculated as (1 - superimposed twitch/resting twitch) $\times 100 \%$ [15]. Root mean square amplitudes of biceps and brachioradialis EMG were measured for $100 \mathrm{~ms}$ prior to motor point stimulation during MVCs. For each set of five MVCs, means of each variable were calculated.

Additional measurements were taken from baseline resting twitches; including twitch onset latency (onset of torque after motor point stimulation), time-to-peak (time from onset to peak torque), and half relaxation time (time from peak torque to time at which the resting twitch decreased by $50 \%$ ).

\section{Blinding}

Participants undertook two conditioning protocols and were unaware that one was a control. The individual who provided verbal encouragement during MVCs was blinded to the conditioning protocol. Measurements of maximal voluntary torque, twitch amplitude, voluntary activation and EMG were performed by a blinded investigator. Once these analyses were performed, and decisions made regarding excluded data (see S3), investigators were unblinded to perform statistical analyses.

\section{Statistics}

Normality of residuals was assessed by visual inspection of histograms and the Shapiro-Wilks test. Homoscedasticity was assessed by visual inspection of residual scatterplots and multicollinearity was assessed with the variance inflation factor (no multicollinearity assumed if variance inflation factor $<5$ ). Assumptions were met except for normality in some cases, outlined below.

Baseline measures on the 2 days were compared using two-tailed, paired $t$ tests (normally distributed data) and Wilcoxon signed-rank tests (non-normal data). For each set after conditioning, averages for each outcome measure were normalised to the baseline average. All outcome measures were expressed as a percentage of baseline except voluntary activation, which was normalised by subtracting baseline from the post-conditioning value.

Linear mixed models were run for each dependent variable (maximal voluntary torque, superimposed twitch amplitude, resting twitch amplitude, voluntary activation, biceps EMG and brachioradialis EMG; all normalised to baseline). Fixed effects for each model were repeated variables of condition (PCMS and control) and time (1 min and $20 \mathrm{~min}$ after), and an unstructured covariance matrix was used. Participants (numbered one to eleven) were a random effect ( $n=11$ for maximal torque and EMG models, $n=9$ for twitch and voluntary activation models). Maximal voluntary torque and superimposed twitch data were non-normal, owing to several outlying data points causing skewed distributions. Normality could be assumed when data points with studentised residuals $> \pm 2$ SD were removed. Linear mixed modelling was performed twice for these variables, once with all data, and once without outlying data points.

To characterise voluntary control of elbow flexors during MVCs in individuals with C4 and C5 spinal cord injuries, median and range were reported for each outcome measure using baseline data from both days averaged for each participant ( $n=11$ for maximal torque and EMG, $n=9$ for twitch and voluntary activation). The same was reported for resting twitch characteristics $(n=9)$.

Multiple linear regression was used to explore the relationship of predictor variables, baseline voluntary activation and the clinical strength assessment, with mean voluntary activation after PCMS $(n=9)$ or control $(n=8)$ conditioning (mean of 1 and $20 \mathrm{~min}$ after; expressed as difference from baseline). To look at individual relationships, simple linear regressions were performed for each predictor variable and the dependent variable (mean voluntary activation after PCMS or control). Multiple and simple linear regressions were also used to explore the relationship of baseline voluntary activation and baseline resting twitch amplitude (predictor variables) with baseline maximal voluntary torque.

Effect sizes (Cohen's $d_{\text {z }}$ ) were calculated for the difference in mean outcome measures (mean of 1 and $20 \mathrm{~min}$ ) after conditioning between PCMS and control protocols [27]. Statistical analyses were performed using IBM SPSS 
Statistics software (version 23). Statistical significance was set at $p<0.05$. Group data are presented as mean (SD) or as median (range).

\section{Results}

\section{Primary analyses}

There were no differences in baseline measures between days for any variable, measured using Wilcoxon signedrank tests (maximal voluntary torque: $p=0.20$; superimposed twitch amplitude: $p=0.64$; voluntary activation: $p$ $=0.89$; biceps EMG: $p=0.58$; brachioradialis EMG: $p=$ 0.65 ) or paired $t$ test (resting twitch amplitude: $p=0.85$ ).

Table 2 shows median and range for each outcome measure using baseline data from both days. For individual baseline data see S4.

Linear mixed modelling on post-conditioning data revealed no effects of condition, time, nor an interaction of condition $\times$ time on maximal voluntary torque, voluntary activation, superimposed twitch amplitude and EMG recorded from biceps and brachioradialis during MVC (all $p$ values $>0.06$; Fig. 2; S5). Resting twitch amplitude also showed no effects of condition $(p=0.44)$ or condition $\times$ time $(p=0.64)$ but did show an effect of time $(p=0.02)$, seen as small decreases in resting twitch amplitude $20 \mathrm{~min}$

Table 2 Median (range) baseline data for each outcome measure using data from both days

\begin{tabular}{|c|c|c|c|}
\hline & \multirow[t]{2}{*}{ Median (range) } & \multicolumn{2}{|c|}{ Historic data } \\
\hline & & Female & Male \\
\hline \multicolumn{4}{|l|}{ Outcome measure } \\
\hline $\begin{array}{l}\text { Maximal voluntary } \\
\text { torque }(\mathrm{Nm})\end{array}$ & $11.4(6.5-41.0)$ & $41.4(6.6)$ & $65.9(8.1)$ \\
\hline $\begin{array}{l}\text { Superimposed twitch } \\
\text { amplitude }(\mathrm{Nm})\end{array}$ & $0.25(0.04-1.24)$ & - & - \\
\hline $\begin{array}{l}\text { Resting twitch } \\
\text { amplitude (Nm) }\end{array}$ & $3.3(2.0-5.8)$ & $6.1(1.6)$ & $10.7(2.0)$ \\
\hline Voluntary activation (\%) & $92.1(62.5-99.4)$ & $97.3(5.1)$ & $95.7(4.1)$ \\
\hline Biceps EMG (mV) & $0.70(0.16-1.95)$ & - & - \\
\hline $\begin{array}{l}\text { Brachioradialis } \\
\text { EMG }(\mathrm{mV})\end{array}$ & $0.27(0.08-0.85)$ & - & - \\
\hline \multicolumn{4}{|l|}{ Resting twitch parameter } \\
\hline Twitch onset & $10.0(8.3-11.2)$ & - & - \\
\hline Time to peak & $86.0(46.7-109.4)$ & - & $65.5(6.2)$ \\
\hline Half relaxation time & $123.6(61.8-169.2)$ & - & $63.3(9.9)$ \\
\hline
\end{tabular}

See S4 for individual baseline data. Historic group data (mean (SD)) are from participants without spinal cord injury. Values for maximal voluntary torque, voluntary activation and resting twitch amplitude are from Allen et al. [31] and values for time to peak and half relaxation time are from McKenzie et al. [18] after both PCMS (96.1\% (10.1) of baseline) and control (92.1\% (8.1) of baseline) conditioning. For the two variables with non-normal distributions, a second model was run with outlying data points excluded. These models similarly showed no effects of condition, time, nor any interactions for maximal voluntary torque or superimposed twitch.

Effect sizes (Cohen's $d_{\mathrm{z}}$ ) for the difference in mean outcome measures (mean of 1 and $20 \mathrm{~min}$ ) after conditioning between PCMS and control protocols were as follows; maximal voluntary torque: 0.05 , superimposed twitch: 0.06 , resting twitch: 0.34 , voluntary activation: 0.28 , biceps EMG: 0.35, brachioradialis EMG: 0.12 .

\section{Exploratory analyses}

Multiple linear regression models indicate that the predictor variables (baseline voluntary activation and the clinical strength assessment) explain $32 \%$ of variance in voluntary activation after PCMS (expressed as difference from baseline) and $26 \%$ of variance after control conditioning (PCMS: $F_{(2,6)}=1.40, p=0.32 ; R^{2}=0.32$; control: $F_{(2,5)}=$ $0.87, p=0.48 ; R^{2}=0.26$ ), however neither variable acts as a significant predictor. Simple linear regression revealed non-significant moderate (as per Mukaka [28]) relationships that were opposite in sign between baseline voluntary activation $(x)$ and voluntary activation after conditioning (difference from baseline; $y)$ on the PCMS day $(R=0.50 ; p$ $=0.17$; equation: $y=-0.14 x+13.00)$ and the control day $(R=0.51 ; p=0.20$; equation: $y=0.14 x-13.28)$. On the PCMS day, lower baseline voluntary activation corresponded to greater increases in voluntary activation after conditioning with the inverse relationship on the control day. Relationships between clinical strength assessment and voluntary activation after conditioning (difference from baseline) were either low or negligible (PCMS: $R=0.13, p$ $=0.73$; control: $R=0.32, p=0.45$ ).

Multiple linear regression of baseline parameters indicates that voluntary activation and resting twitch amplitude together explain $85 \%$ of the variance in baseline maximal voluntary torque $\left(F_{(2,6)}=17.56, p=0.003 ; R^{2}=0.85\right)$. Both variables together predict maximal voluntary torque better than either variable alone. Simple linear regressions revealed a moderate relationship between voluntary activation $(x)$ and maximal voluntary torque $(y)(R=0.67$; equation: $y=0.66 x-38.18$; Fig. 3a) and a high-strength relationship between resting twitch amplitude $(x)$ and maximal voluntary torque $(y)(R=0.87$; equation: $y=$ $7.53 x-6.88$; Fig. $3 b$ ), whereby higher baseline voluntary activation or resting twitch amplitudes corresponded to higher baseline maximal voluntary torque. S6 contains coefficients for each regression model and associated $t$ statistics and $p$ values. 

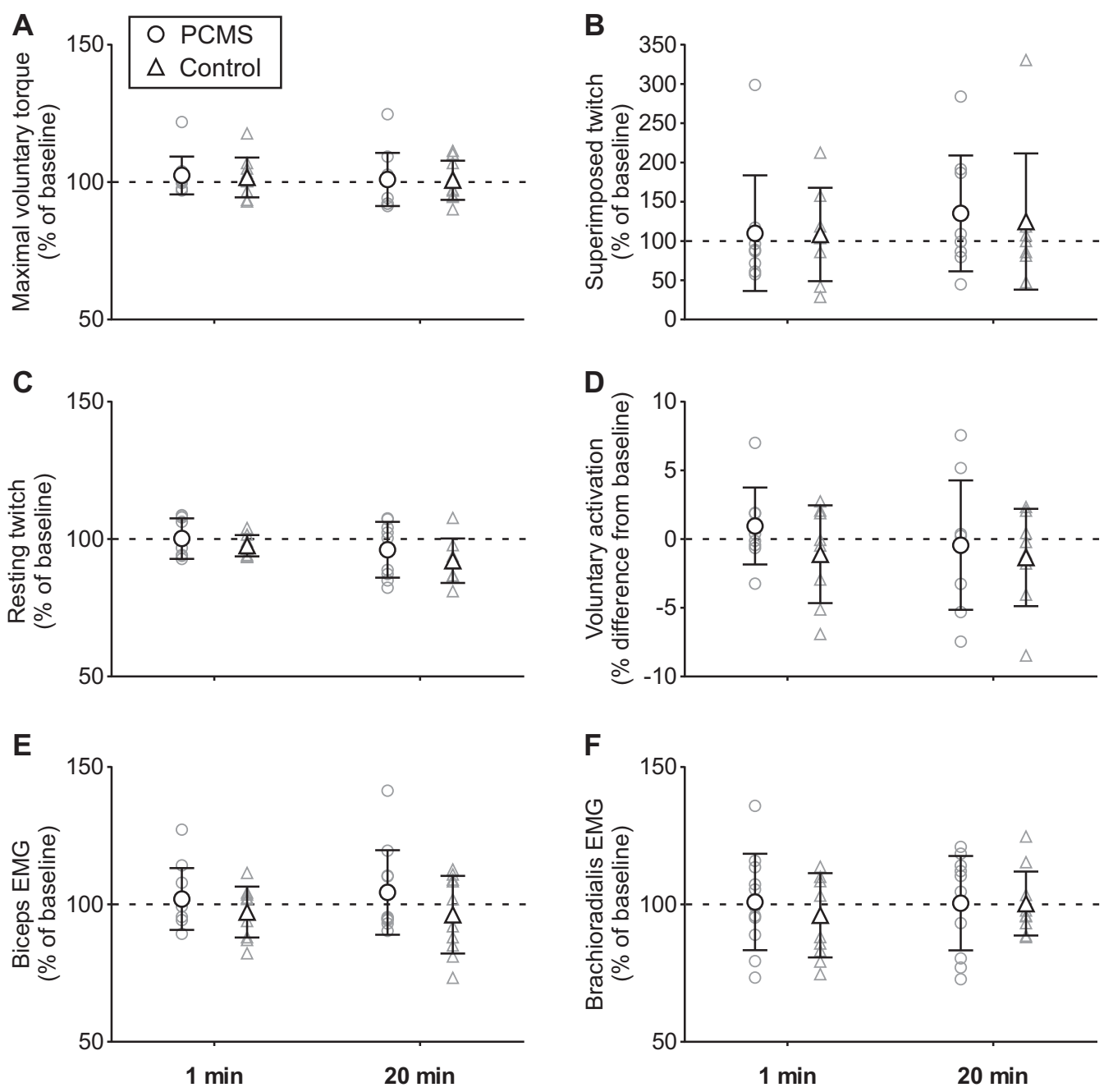

Fig. 2 Group data (black symbols are mean (SD), smaller grey symbols are individual data) showing maximal voluntary torque a, superimposed $\mathbf{b}$ and resting twitch $\mathbf{c}$ amplitudes, maximal voluntary activation $\mathbf{d}$ and maximal biceps e and brachioradialis $\mathbf{f}$ EMG after paired corticospinal-motoneuronal stimulation (PCMS; O) or control

\section{Discussion}

Here we showed that PCMS had no significant effects on maximal torque, voluntary activation or muscle activity during maximal voluntary elbow flexion in a group of individuals with cervical spinal cord injury. Thus, the hypothesis was not supported by the results of this study.

By contrast to results seen here, previous work shows that PCMS can increase biceps potentials and enhance voluntary motor output during low-force elbow flexions [79], however these studies only tested for changes in corticospinal transmission to lower-threshold motoneurones supplying the biceps, and PCMS may be less effective at modifying transmission to the higher-threshold motoneurones that were tested in the current study. The only other study to test MVCs after PCMS showed enhanced maximal

$(\triangle)$ conditioning. Individual data points are averages for each set of five maximal voluntary contractions, normalised to baseline. There were no significant differences between PCMS and control conditioning for any outcome measure

voluntary activation, indicating that high-threshold motoneurones can be affected by PCMS. However, this was for the adductor pollicis muscle in people without spinal cord injury [11], whereas here we targeted the biceps brachii in people with spinal cord injury. The corticospinal pathway has a large capacity for plasticity after spinal cord injury [1], and PCMS is shown to enhance corticospinal transmission in people with spinal cord injury $[6,10]$. Therefore, the critical difference between studies may be the different muscles tested. In people without a spinal cord injury, voluntary activation of thumb adductors $(\sim 90 \%)[11,29]$ is lower than elbow flexors ( 95-99\%) [18, 30-32], so there may be more room for improvement for thumb adduction. Here, median baseline voluntary activation was $92 \%$, with several participants activating at similar levels to those without injury. Those with high voluntary activation might 
A

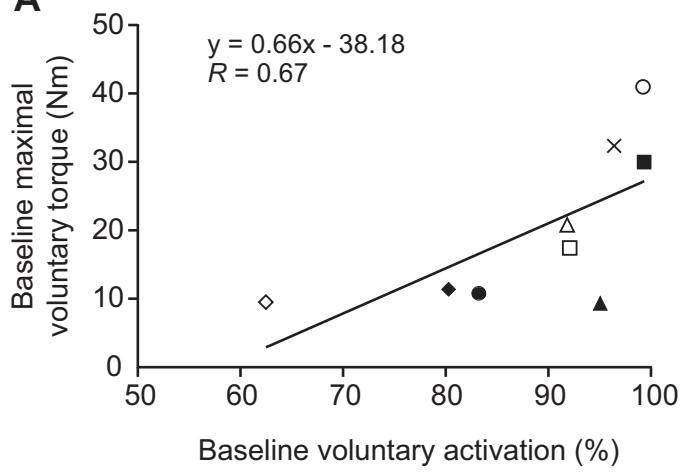

B

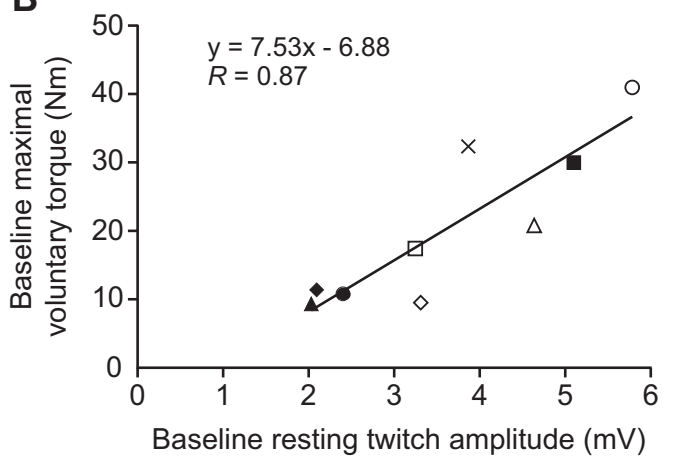

Fig. 3 Regression analyses exploring the relationships of baseline voluntary activation $\mathbf{a}$ and baseline resting twitch amplitude $\mathbf{b}$ with baseline maximal voluntary torque. Baseline data are mean values from both days of the study. Each point represents data for a single individual, and each participant is represented by the same unique symbol in both graphs. There was a moderate relationship between voluntary activation and maximal voluntary torque $(p=0.047)$ and a high-strength relationship between resting twitch amplitude and maximal voluntary torque $(p=0.002)$, whereby higher baseline voluntary activation or resting twitch amplitudes corresponded to higher baseline maximal voluntary torque. Regression equations and correlation coefficients $(R)$ are shown for each graph

have little room for improvement. Thus, a ceiling effect of elbow flexor voluntary activation may have contributed to the current results. Although not significant, the relationship of lower baseline voluntary activation corresponding to greater increases in voluntary activation after PCMS supports this idea.

Another reason that PCMS might be less effective for biceps than adductor pollicis is that hand muscles are innervated by a greater proportion of motoneurones arising from the direct monosynaptic pathway, whereas more proximal muscles (such as the biceps) have a greater contribution from oligosynaptic pathways [3]. PCMS is designed to target the monosynaptic pathway, therefore, it may be more effective for adductor pollicis than for the more proximal biceps muscle. This idea is supported by previous studies, which show greater rates of PCMSinduced facilitation when a hand muscle is targeted than when biceps is targeted [6-8]. In addition, although the extent of spinal damage is unknown in this group of participants, motoneurone death tends to be greatest closer to the lesion epicentre [33]. Therefore, distal muscles that are innervated caudal to the spinal injury level may be less affected by motoneurone death and may have a greater number of intact corticospinal-motoneuronal connections available for PCMS to target.

Another limitation of the study is that the contribution of other muscles to elbow flexion was unexplored. Although triceps brachii activity was not assessed here, it is possible that elbow extensor coactivation could have interfered with elbow flexion torque measurement. The contribution of an important elbow flexor, brachialis, was also not assessed, although given the muscles' proximity and a common innervation by the musculocutaneous nerve, it is likely that brachialis and biceps would have responded similarly to PCMS.

Plasticity-inducing protocols in humans are well known for high levels of variability arising from numerous sources, including age, time-of-day, previous activity, attentional state and genetic polymorphisms [34]. Here we accounted for time-of-day by keeping session start-times consistent, however we cannot rule out other variability sources. The TMS component of PCMS is a source of variability, given that each stimulus elicits multiple descending volleys [35]. The earliest volley is used to calculate ISIs here, therefore later volleys would arrive simultaneously with or after the postsynaptic, motoneuronal potential, possibly counteracting the effects of the first volley to result in no change, or in depression. Similarly, although PCMS targets corticospinal-motoneuronal synapses [7-9], changes at other locations cannot be ruled out, and the order of inputs at these locations would be different. Other parameters that may influence responses to PCMS are TMS intensity and the number of stimulus pairs delivered for conditioning [8]. Despite numerous sources of variability, PCMS is reliable day-to-day in those who do respond in facilitation [8], thus screening for facilitation may be an important step to identify individuals for whom the technique is likely to be effective. Such screening may be particularly important for a spinal cord injured population where cortical and spinal circuitry changes are complex and highly individualised, making the precise effects of PCMS difficult to determine, especially when the targeted corticospinal-motoneuronal synapses are at or near the lesion's epicentre.

Although PCMS had no effect, this study has further characterised the effects of cervical spinal cord injury on motor output during maximal elbow flexion. Similarly to our study, previous studies in muscle groups other than elbow flexors show large reductions in force during maximal isometric contractions after incomplete spinal cord injury $(\sim 10-75 \%$ of forces produced by people without injury) [6, 10, 19-21, 23, 24]. Contrasting our results however, these reductions in force were accompanied by 
large reductions in voluntary activation for knee extensors, ankle plantar flexors and wrist flexors $(\sim 50-75 \%$ of activation levels produced by people without injury) $[19,20,24]$. To our knowledge, no study has previously assessed biceps maximal voluntary activation after cervical spinal cord injury, thus it is interesting to see that while maximal torque (median $11 \mathrm{Nm}$ for males and $21 \mathrm{Nm}$ in the single female) was greatly reduced in comparison with people without a spinal cord injury $(65-70 \mathrm{Nm}$ in males and $40-45 \mathrm{Nm}$ in females) $[18,30-32]$, maximal voluntary activation remained relatively high.

Muscle weakness after spinal cord injury can result from decreased corticospinal input to motoneurones [1], a reduced number of intact motoneurones [33] or to peripheral changes in the muscle [36]. Here, moderate-to-high strength relationships of both baseline voluntary activation and baseline resting twitch amplitude with baseline maximal voluntary torque suggest that both central and peripheral factors contributed to strength in this group of individuals. For several participants in this study, the combination of high voluntary activation with low torque suggests that such individuals are weak because they have lost a proportion of the motoneurones that innervate biceps. This is consistent with the location of their spinal injuries close to the biceps motoneurone pool (C5-7). Although these individuals may also have reduced corticospinal input to biceps motoneurones, what remains is sufficient to activate remaining motoneurones optimally. The weakness of individuals with low baseline voluntary activation is likely owing to inadequate corticospinal input. Hence, PCMS might be more effective, and future studies may benefit from screening for such individuals. In general, muscles with motoneurone pools more caudal to the injury site are likely to be better targets for this intervention.

Here we also characterised biceps contractile properties in response to single, suprathreshold stimulation (resting twitch amplitude and latencies). Spinal cord injury often results in muscle atrophy and transformation of muscle fibres to predominantly fast-twitch, type-II glycolytic fibres, causing decreases in force output, faster contractile properties and lower fatigue resistance [36]. As expected, baseline potentiated resting twitch amplitudes were lower here than in control samples using the same motor point stimulation and elbow angle [18, 31]. However, time-topeak and half relaxation time were slower (time-to-peak: $86 \mathrm{~ms}$; half relaxation: $124 \mathrm{~ms}$ ) than in controls (time-topeak: $\sim 65 \mathrm{~ms}$; half relaxation: $\sim 70 \mathrm{~ms})[18,37,38]$. These slower, rather than faster, contractile properties may reflect a difference in proximity of injury to the relevant motoneurone pool. Here, the location of injury was close to the biceps motoneurone pool in all participants, resulting in an increased likelihood of biceps motoneurone death and denervation [33], whereas previous studies have tested lower limb muscles with motoneurone pools that were often more caudal to the lesion's epicentre [36]. Results here are similar to a study of the thenar muscles, which showed non-significant slowing of time-to-peak and half relaxation time after spinal cord injury in comparison to people without injury [39].

In conclusion, there was no effect of PCMS on maximal voluntary motor output of the elbow flexors in this group of individuals with cervical spinal cord injury. Results could be explained by a ceiling effect for voluntary activation or PCMS may be more effective at targeting corticospinal transmission to lower-threshold motoneurones supplying elbow flexors, or to motoneurones supplying more distal muscles. Despite the lack of effect, PCMS is previously shown to enhance corticospinal transmission to several muscles and improve motor function in people with and without spinal cord injury. Thus, further studies are warranted to examine the full potential of the technique.

\section{Data archiving}

Individual data are presented as supplementary information (see S7).

Acknowledgements We acknowledge Dr James Nuzzo, Zoë Djajadikarta, Harrison Finn and Dr Euan McCaughey for their assistance during experiments, and acknowledge participants for their contributions to the study.

Funding This study was supported by a Program Grant (1055084) from the National Health and Medical Research Council of Australia (NHMRC).

Author contributions SCD and JLT conceived the work, and SCD, CLBR, JEB and JLT designed the work. SCD and CLBR acquired data and SCD, CLBR, JEB and JLT played an important role in interpreting the results. SCD drafted the manuscript and SCD, CLBR, JEB and JLT revised the manuscript. SCD, CLBR, JEB and JLT approved the final version and agreed to be accountable for all aspects of the work in ensuring that questions related to the accuracy or integrity of any part of the work are appropriately investigated and resolved.

\section{Compliance with ethical standards}

Conflict of interest The authors declare no conflicts of interest.

Statement of ethics We certify that all applicable institutional and governmental regulations concerning the ethical use of human volunteers were followed during the course of this research. Procedures were approved by the Human Research Ethics Committee of the University of New South Wales (approval number HC12556). The study was conducted according to the Declaration of Helsinki but was not registered in a publicly accessible database prior to participant recruitment. 
Publisher's note: Springer Nature remains neutral with regard to jurisdictional claims in published maps and institutional affiliations.

\section{References}

1. Oudega M, Perez MA. Corticospinal reorganization after spinal cord injury. J Physiol. 2012;590:3647-63.

2. de Noordhout AM, Rapisarda G, Bogacz D, Gérard P, De Pasqua V, Pennisi G, et al. Corticomotoneuronal synaptic connections in normal man: an electrophysiological study. Brain. 1999;122: 1327-40.

3. Palmer E, Ashby P. Corticospinal projections to upper limb motoneurones in humans. J Physiol. 1992;448:397-412.

4. Rathelot J-A, Strick PL. Subdivisions of primary motor cortex based on cortico-motoneuronal cells. Proc Natl Acad Sci. 2009; 106:918-23.

5. Feldman Daniel E. The spike-timing dependence of plasticity. Neuron. 2012;75:556-71.

6. Bunday KL, Perez MA. Motor recovery after spinal cord injury enhanced by strengthening corticospinal synaptic transmission. Curr Biol. 2012;22:2355-61.

7. Dongés SC, D'Amico JM, Butler JE, Taylor JL. The involvement of N-methyl-D-aspartate receptors in plasticity induced by paired corticospinal-motoneuronal stimulation in humans. J Neurophysiol. 2018;119:652-61.

8. Fitzpatrick SC, Luu BL, Butler JE, Taylor JL. More conditioning stimuli enhance synaptic plasticity in the human spinal cord. Clin Neurophysiol. 2016;127:724-31.

9. Taylor JL, Martin PG. Voluntary motor output is altered by spiketiming-dependent changes in the human corticospinal pathway. J Neurosci. 2009;29:11708-16.

10. Urbin MA, Ozdemir RA, Tazoe T, Perez MA. Spike-timingdependent plasticity in lower-limb motoneurons after human spinal cord injury. J Neurophysiol. 2017;118:2171-80.

11. D'Amico JM, Donges SC, Taylor JL. Paired corticospinalmotoneuronal stimulation increases maximal voluntary activation of human adductor pollicis. J Neurophysiol. 2017;119:369-76.

12. Shield A, Zhou S. Assessing voluntary muscle activation with the twitch interpolation technique. Sports Med. 2004;34:253-67.

13. Bellemare F, Bigland-Ritchie B. Assessment of human diaphragm strength and activation using phrenic nerve stimulation. Respir Physiol. 1984;58:263-77.

14. Merton PA. Voluntary strength and fatigue. J Physiol. 1954;123: 553-64.

15. Allen GM, Gandevia SC, McKenzie DK. Reliability of measurements of muscle strength and voluntary activation using twitch interpolation. Muscle Nerve. 1995;18:593-600.

16. Behm DG, Whittle J, Button D, Power K. Intermuscle differences in activation. Muscle Nerve. 2002;25:236-43.

17. Gandevia SC, Allen GM, Butler JE, Taylor JL. Supraspinal factors in human muscle fatigue: evidence for suboptimal output from the motor cortex. J Physiol. 1996;490:529-36.

18. McKenzie DK, Bigland-Ritchie B, Gorman RB, Gandevia SC. Central and peripheral fatigue of human diaphragm and limb muscles assessed by twitch interpolation. J Physiol. 1992;454: 643-56.

19. Jayaraman A, Gregory CM, Bowden M, Stevens JE, Shah P, Behrman AL, et al. Lower extremity skeletal muscle function in persons with incomplete spinal cord injury. Spinal Cord. 2006; $44: 680$
20. Kim HE, Thompson CK, Hornby TG. Muscle activation varies with contraction mode in human spinal cord injury. Muscle Nerve. 2015;51:235-45.

21. Thomas CK, Tucker ME, Bigland-Ritchie B. Voluntary muscle weakness and co-activation after chronic cervical spinal cord injury. J Neurotrauma. 1998;15:149-61.

22. Needham-Shropshire MB, Klose KJ, Tucker M, Thomas C. Manual muscle test score and force comparisons after cervical spinal cord injury. J Spinal Cord Med. 1997;20:324-30.

23. Cremoux S, Amarantini D, Tallet J, Dal Maso F, Berton E. Increased antagonist muscle activity in cervical SCI patients suggests altered reciprocal inhibition during elbow contractions. Clin Neurophysiol. 2016;127:629-34.

24. Lin K-H, Chen Y-C, Luh J-J, Wang C-H, Chang Y-J. H-reflex, muscle voluntary activation level, and fatigue index of flexor carpi radialis in individuals with incomplete cervical cord injury. Neurorehabil Neural Repair. 2012;26:68-75.

25. Bunday KL, Oudega M, Perez MA. Aberrant crossed corticospinal facilitation in muscles distant from a spinal cord injury. PLOS ONE. 2013;8:e76747.

26. Kendall FP, McCreary EK, Provance PG, Rodgers MM, Romani WA. Muscles: Testing and Function, with Posture and Pain. 5th edn. Philadelphia: Lippincott Williams and Wilkins; 2005.

27. Faul F, Erdfelder E, Lang A-G, Buchner A. G*Power 3: A flexible statistical power analysis program for the social, behavioral, and biomedical sciences. Behav Res Methods. 2007;39:175-91.

28. Mukaka MM. A guide to appropriate use of correlation coefficient in medical research. Malawi Med J. 2012;24:69-71.

29. Herbert RD, Gandevia SC. Muscle activation in unilateral and bilateral efforts assessed by motor nerve and cortical stimulation. J Appl Physiol. 1996;80:1351-6.

30. Allen GM, Gandevia AS, Middleton J. Quantitative assessments of elbow flexor muscle performance using twitch interpolation in post-polio patients: no evidence for deterioration. Brain. 1997; 120:663-72.

31. Allen GM, Gandevia SC, Neering IR, Hickie I, Jones R, Middleton J. Muscle performance, voluntary activation and perceived effort in normal subjects and patients with prior poliomyelitis. Brain. 1994;117:661-70.

32. Kennedy DS, McNeil CJ, Gandevia SC, Taylor JL. Fatiguerelated firing of distal muscle nociceptors reduces voluntary activation of proximal muscles of the same limb. J Appl Physiol. 2014;116:385-94

33. Grumbles RM, Thomas CK. Motoneuron death after human spinal cord injury. J Neurotrauma. 2017;34:581-90.

34. Ridding MC, Ziemann U. Determinants of the induction of cortical plasticity by non-invasive brain stimulation in healthy subjects. J Physiol. 2010;588:2291-304.

35. Di Lazzaro V, Oliviero A, Pilato F, Mazzone P, Insola A, Ranieri F, et al. Corticospinal volleys evoked by transcranial stimulation of the brain in conscious humans. Neurol Res. 2003;25:143-50.

36. Biering-Sørensen B, Kristensen IB, Kjær M, Biering-Sørensen F Muscle after spinal cord injury. Muscle Nerve. 2009;40:499-519.

37. Bowden JL, Taylor JL, McNulty PA. Voluntary activation is reduced in both the more- and less-affected upper limbs after unilateral stroke. Front Neurol. 2014;5:239.

38. Lampropoulou SI, Nowicky AV, Marston L. Magnetic versus electrical stimulation in the interpolation twitch technique of elbow flexors. J Sports Sci Med. 2012;11:709-18.

39. Thomas CK. Contractile properties of human thenar muscles paralyzed by spinal cord injury. Muscle Nerve. 1997;20: $788-99$. 